\title{
Analysis of the learning curve for beating heart, totally endoscopic, coronary artery bypass grafting
}

\author{
Nan Cheng, MD, Changqing Gao, MD, Ming Yang, MD, Yang Wu, MD, Gang Wang, MD, and \\ Cangsong Xiao, MD
}

\begin{abstract}
Background: Robotic cardiac surgery has been proved safe and feasible in dedicated centers. We systematically analyzed the learning curve issues associated with totally endoscopic coronary artery bypass grafting (TECAB) using a stepwise approach by a single surgeon who had successfully performed $>650$ cases of various types of robotic cardiac surgery at our single center.
\end{abstract}

\begin{abstract}
Methods: From January 2007 to March 2013, 230 patients underwent robotic coronary bypass grafting on the beating heart. Of these patients, 90 had successfully undergone beating heart TECAB using the da Vinci S/Si Surgical System without conversion to sternotomy. All beating heart TECAB procedures were completed using the following modules: endoscopic left internal thoracic artery (LITA) harvesting, pericardiotomy and target vessel identification, and anastomosis of the LITA to the target vessel. The perioperative outcomes were compared among 3 quintiles of 30 consecutive patients each and the learning curve results were evaluated.

Results: No in-hospital mortality or severe morbidity occurred. The comparison among the 3 quintiles showed a significant decrease in operative time $(P=.000)$, LITA harvesting time $(P=.037)$, and anastomotic time $(P=.000)$. A significant learning curve was observed for the operative time $[\mathrm{y}(\mathrm{min})=223-17 \times \ln (\mathrm{x})$; $\left.\mathrm{r}^{2}=0.217, P=.000\right]$; LITA harvesting time $\left[\mathrm{y}(\min )=37-3 \times \ln (\mathrm{x}) ; \mathrm{r}^{2}=0.097, P=.003\right] ;$ and LITA-left anterior descending artery anastomotic time $\left[\mathrm{y}(\mathrm{min})=18-2 \times \ln (\mathrm{x}) ; \mathrm{r}^{2}=0.298, P=.000\right]$. No differences were found in the mean transit flow $(P=.102)$ or perioperative complications among the 3 quintiles.

Conclusions: Modular-based TECAB procedures can be successfully performed; however, each module has a steep learning curve. A stable and well-trained robotic cardiac team and an experienced cardiac surgeon can achieve good, reproducible results after this substantial learning curve. (J Thorac Cardiovasc Surg 2014;148:1832-6)
\end{abstract}

Robotic technology has enabled cardiac surgeons to perform closed-chest, off-pump coronary artery bypass grafting. Just as with any new surgical procedure, a learning curve will be inevitable for totally endoscopic coronary artery bypass grafting (TECAB). Since the first successful TECAB on an arrested heart was reported by Loulmet and colleagues, ${ }^{1}$ the TECAB technique has been proved safe, feasible, and reproducible. With the introduction of the endoscopic stabilizer, many centers are currently able to perform both endoscopic harvesting of the internal thoracic arteries and coronary anastomoses using anastomotic clips during beating heart $(\mathrm{BH})$ TECAB. Since our first TECAB in January 2007, we have performed

From the Department of Cardiovascular Surgery, Chinese People's Liberation Army General Hospital, Beijing, China.

Disclosures: Authors have nothing to disclose with regard to commercial support. Received for publication Dec 11, 2013; revisions received Jan 21, 2014; accepted for publication Feb 4, 2014; available ahead of print March 12, 2014.

Address for reprints: Changqing Gao, MD, Department of Cardiovascular Surgery, Chinese People's Liberation Army General Hospital, No 28, Fuxing Rd, Haidian District, Beijing 100853, China (E-mail: gchq301@yahoo.com).

$0022-5223 / \$ 36.00$

Copyright $($ c 2014 by The American Association for Thoracic Surgery

http://dx.doi.org/10.1016/j.jtcvs.2014.02.002
$>650$ cases of robotic cardiac surgery, comprising several different types of robotic procedures performed by a single surgeon. ${ }^{2-4}$ However, learning curve issues will be involved for all types of robotic surgery. Therefore, we aimed to analyze our team's progress with the development of TECAB by examining different quintiles and evaluating the learning curve results for our single team and single surgeon using a stepwise approach.

\section{METHODS}

From January 2007 to March 2013, 650 robotic cardiac operations using the da Vinci S/Si Surgical System (Intuitive Surgical, Inc, Sunnyvale, Calif) were performed. The institutional review board of our institution approved the present study, and all patients provided written informed consent. Of these 650 patients, 230 underwent robotic-assisted coronary artery bypass grafting on the $\mathrm{BH}$. Of these 230 patients, 90 (70 men and 20 women) underwent $\mathrm{BH}$ TECAB. The mean age of the patients who underwent BH TECAB was $59.1 \pm 10.2$ years (range, 33-80). The preoperative data are listed in Table 1.

\section{Patient Selection}

Patients whose coronary angiogram showed total occlusion or significant $(\geq 75 \%)$ stenosis in the left anterior descending artery (LAD) with or without non-LAD lesions that were amenable for percutaneous coronary intervention angioplasty were enrolled in the present study. 


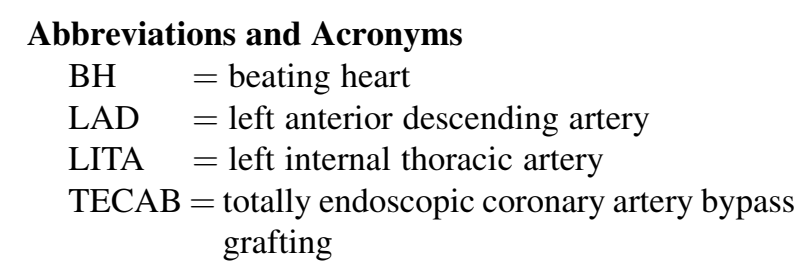

The patients selected for BH TECAB were assessed preoperatively by $\geq 2$ senior cardiologists.

The exclusion criteria were a history of left lung trauma or surgery, a left ventricular ejection fraction of $<30 \%$, a requirement for emergency coronary artery bypass grafting, and the presence of severe chronic obstructive pulmonary disease with an inability to tolerate 1-lung ventilation.

\section{Anesthesia and Preparation}

After the routine establishment of anesthesia and relaxation, doublelumen intubation was performed for 1-lung ventilation. Percutaneous defibrillator patches were used, and the patient was placed in a $30^{\circ}$ right lateral decubitus position. A camera port was introduced into the left hemithorax through the incision in the fifth intercostal space on the anterior axillary line under left lung collapse. Carbon dioxide was insufflated and maintained at a target of 6 to $12 \mathrm{~mm} \mathrm{Hg}$, with a stable hemodynamic status. Instrument ports were then introduced through the third and seventh intercostal spaces on the mammary line under endoscopic vision.

\section{Endoscopic Left Internal Thoracic Artery Harvesting}

The endothoracic fascia was removed to adequately visualize the left internal thoracic artery (LITA). The LITA was completely harvested from the subclavian vein to the LITA bifurcation in a totally skeletonized fashion. Electrocautery and endoscopic clips were used during division of the pedicle side branches. In some cases, the LITA was harvested with the accompanying veins, because the arteries and veins were too close in proximity.

\section{Pericardiotomy and Target Vessel Identification}

The LITA was attached to the endothoracic connecting areolar tissue to prevent it from hanging over the pericardium. The pericardial fat pad was removed by electrocautery, and an incision of the pericardium from the sternal border nearly to the apex of the left ventricle was created. The target vessel was identified by a $30^{\circ}$ downward camera angle.

\section{Anastomosis of LITA to Target Vessel}

A 12-mm cannula was introduced into the thoracic cavity as the fourth port at the subcostal margin, close to the midclavicular line. The Endowrist Stabilizer (Intuitive Surgical, Sunnyvale, Calif) was inserted through the fourth port. The patient was heparinized, and the LITA was transected for anastomosis. The saddle loops were placed proximally and distally around the LAD, and about $8 \mathrm{U}$-Clips (Medtronic, Minneapolis, Minn) were used to interruptedly parachute the LITA onto the LAD. The blood flow of the LITA was measured using transit-time ultrasonography with a flexible flow probe (Medistim, Inc, Oslo, Norway).

\section{Intraoperative Conversion}

Conversions to a small anterior thoracotomy approach were performed for technical or anastomotic reasons during the TECAB procedure, such as dense pleural adhesions, an intramyocardial coronary target, LITA injury, an unsatisfactory anastomosis, or hemodynamic instability with carbon dioxide insufflation.

\section{Postoperative Graft Patency Assessment and Follow-up}

Angiography was performed before discharge to evaluate graft patency and LITA-LAD anastomosis. Those who could not tolerate, or refused to undergo angiography, underwent 64-slice spiral computed tomography angiography. After discharge, all patients were followed up by computed tomography angiography.

\section{Statistical Analysis}

Statistical analysis was performed using the Statistical Package for Social Sciences, version 13.0 (SPSS, Inc, Chicago, Ill). Continuous variables are presented as the mean \pm standard deviation and categorical variables as absolute values and percentages. Statistical significance was based on 2 -sided $P$ values of $<.05$. The 90 patients who underwent TECAB were divided into 3 quintiles of 30 consecutive patients each. Analysis of variance was used to compare the operative and postoperative data of each 30-patient quintile. Regression models with a logarithmic curve fit were applied for the learning curve analysis.

\section{RESULTS}

No major intraoperative technical failures occurred with the da Vinci S/Si system. A total of 230 patients underwent selective robotic-assisted coronary bypass grafting on the $\mathrm{BH}$. Of these, 90 patients underwent TECAB with single LITA-to-LAD anastomoses, and 140 patients required a small minithoracotomy to facilitate the coronary anastomosis. No conversions to sternotomy because of hemodynamic decompensation or anastomotic bleeding were necessary. No patient experienced myocardial infarction postoperatively.

The intraoperative data are listed in Table 2. The total operative time, LITA harvesting time, anastomotic time, mean transit blood flow, and early postoperative data in each 30-patient quintile are listed in Table 3. A substantial decrease occurred from the first to third quintile in the total operative time $(P=.000)$, harvesting time $(P=.037)$, and anastomotic time $(P=.000)$. No clinical or statistically significant differences were found among the quintiles in the mean transit blood flow, intensive care unit stay, mechanical ventilation time, chest drainage volume, or packed red blood cell volume $(P>.05)$. One patient underwent reoperation for bleeding, one developed pneumonia, and one developed a minor stroke. All 3 of these patients were discharged with satisfactory outcomes.

Figure 1 shows a statistically significant learning curve pattern among the 230 patients for the LITA harvesting time $\left[\mathrm{y}(\min )=37-3 \times \ln (\mathrm{x}), \mathrm{r}^{2}=0.097, P=.003\right]$, complete TECAB operative time $[\mathrm{y}(\min )=223-17 \times \ln (\mathrm{x})$, $\left.\mathrm{r}^{2}=0.217, P=.000\right]$, and LITA-to-LAD anastomotic time $\left[\mathrm{y}(\min )=18-2 \times \ln (\mathrm{x}), \mathrm{r}^{2}=0.298, P=.000\right]$, with $\mathrm{y}$ indicating (1) in LITA harvesting time, the LITA takedown time and $\mathrm{x}$, the consecutive LITA takedown number (230 cases) and (2) in TECAB operative time and LITA-LAD anastomotic time, the operative time or anastomotic time, and $\mathrm{x}$, the consecutive TECAB number (90 cases). In contrast, no significant reduction in the mean transit blood flow 
TABLE 1. Patient demographics and preoperative characteristics $(\mathbf{n}=90)$

\begin{tabular}{lc}
\hline \multicolumn{1}{c}{ Variable } & Value \\
\hline Gender & \\
$\quad$ Male & $70(77)$ \\
Female & $20(22)$ \\
Smoking & $43(48)$ \\
Diabetes & $23(26)$ \\
Hypercholesterolemia & $17(19)$ \\
Arterial hypertension & $48(53)$ \\
Cerebrovascular accident & $3(3)$ \\
Chronic lung disease & $2(2)$ \\
Peripheral vascular disease & $1(1)$ \\
History of myocardial infarction & $13(14)$ \\
History of PCI & $9(10)$ \\
Number of diseased coronary vessels $\geq 2$ & $39(43)$ \\
Age (y) & $59.1 \pm 10.2$ \\
Weight (kg) & $69.3 \pm 10.9$ \\
LVEF $(\%)$ & $62.9 \pm 6.7$ \\
\hline Da &
\end{tabular}

Data presented as $\mathrm{n}(\%)$ or mean \pm standard deviation. $P C I$, Percutaneous intervention; $L V E F$, left ventricular ejection fraction.

$\left(\mathrm{r}^{2}=0.024, P=.143\right)$ was noted among the different quintiles.

The average follow-up time was $37.6 \pm 15.5$ months (range, 1-79). The angiographic or computed tomography angiograms showed the graft patency was $100 \%$. Two patients had about $50 \%$ stenosis in the anastomotic site, and 1 patient had low density in the LITA graft on the follow-up computed tomography scan. No major adverse cardiac events occurred during their eligible 3-year follow-up period.

\section{DISCUSSION}

Since the first TECAB procedure was performed by Loulmet and colleagues ${ }^{1}$ in 1998, it has become a safe and feasible procedure for coronary revascularization in many centers. Although it has been criticized for its prolonged operative time compared with open procedures, the adoption of a stepwise approach for such a complex procedure as TECAB has ensured high success rates, reaching $80 \%{ }^{5}$ We have divided our procedure into the following modules: LITA harvesting, pericardial lipectomy and pericardiotomy, and LITA-to-LAD anastomosis. Because of these divisions, TECAB has evolved into more complex procedures, such as the use of the bilateral intrathoracic arteries and hybrid revascularization. Since 2007, our institution has insisted on performing $\mathrm{BH}$

TABLE 2. Intraoperative outcomes

\begin{tabular}{lr}
\hline \multicolumn{1}{c}{ Outcome } & Mean \pm SD \\
\hline LITA takedown time $(\mathrm{min})$ & $25.6 \pm 9.9$ \\
LITA to target vessel robotically anastomotic time (min) & $9.6 \pm 4.1$ \\
Mean operative time (min) & $152.3 \pm 38.6$ \\
Mean transit blood flow (mL/min) & $35.6 \pm 20.7$ \\
\hline SD, Standard deviation; LITA, left internal thoracic artery. &
\end{tabular}

TABLE 3. Perioperative outcomes in each 30-patient quintile

\begin{tabular}{lcccc}
\hline \multicolumn{1}{c}{ Variable } & Quintile 1 & Quintile 2 & Quintile 3 & $\begin{array}{c}\boldsymbol{P} \\
\text { value }\end{array}$ \\
\hline Operative time (min) & $179.0 \pm 36$ & $157.6 \pm 30$ & $145.9 \pm 28$ & .000 \\
LITA harvest time (min) & $29.6 \pm 10$ & $25.3 \pm 9$ & $23.4 \pm 9$ & .037 \\
Anastomotic time (min) & $12.1 \pm 5$ & $8.8 \pm 3$ & $7.8 \pm 3$ & .000 \\
Mean transit blood flow & $29.5 \pm 19$ & $36.5 \pm 21$ & $40.8 \pm 20$ & .102 \\
$\quad(\mathrm{~mL} / \mathrm{min})$ & & & & \\
ICU stay (h) & $27.2 \pm 20$ & $25.4 \pm 21$ & $26.4 \pm 20$ & .789 \\
Intubation time (h) & $7.2 \pm 2$ & $6.7 \pm 2$ & $6.4 \pm 2$ & .246 \\
Chest drainage (mL) & $417.3 \pm 240$ & $430.9 \pm 180$ & $453.27 \pm 240$ & .821 \\
PRBC transfusion (U) & $0.82 \pm 2$ & $0.77 \pm 1$ & $1.05 \pm 2$ & .782 \\
\hline Data presented as mean \pm standard deviation. LITA, Left internal thoracic artery; \\
ICU, intensive care unit; PRBC, packed red blood cell.
\end{tabular}

TECAB, and these modules have been reproducibly performed in $>200$ cases.

The learning curve remains a sensitive subject in the evaluation of new procedure performance, not only in the overall surgical course, but also in each module. After a steep slope with the first 18 to 20 patients, the learning curve became moderate, ${ }^{6}$ and apart from human factors and surgical errors, this slope might precisely reflect the improvement in surgical performance for a dedicated cardiac surgery team. Wiedemann and colleagues ${ }^{7}$ noted a reduction in the LITA takedown times from almost 80 minutes at the beginning of TECAB to approximately 20 minutes. Srivastava and colleagues ${ }^{8}$ described similar results in the mean LITA takedown times, reporting an approximately 34-minute reduction for 164 completed TECAB cases. Schachner and colleagues ${ }^{9}$ also experienced LITA takedown times that were comparable with our results. Oehlinger and colleagues ${ }^{10}$ reported a robotic LITA harvesting time that decreased from 140 minutes in the first 10 cases to 34 minutes in the last 10 cases. Also, they reported 3 cases of LITA injury in the first half of the experience (50 cases), and only 1 case during the second half. Our learning curve results are comparable with those described by these groups. In the present study, the target LITA harvesting speed was achieved within a 30-minute range in the third quintile.

Considering the difficulties in a magnified, bouncing operative field, anastomoses during BH TECAB will be more challenging than in arrested heart TECAB. The introduction of a suction stabilizer inserted into the thoracic cavity that is controlled by the operator from the console can be regarded as a significant step in TECAB development. The robotic LITA-to-LAD anastomotic times reported in previous studies were within the range of 13 to 35 minutes. ${ }^{5,7,8,11}$ Using U-clips and stabilizers, we have almost achieved the ultimate goal of minimally invasive coronary operations (free of groin incision, no cardiac arrest, and no need for cardiopulmonary bypass) with anastomotic times slightly shorter than those previously reported. One patient underwent conversion to 


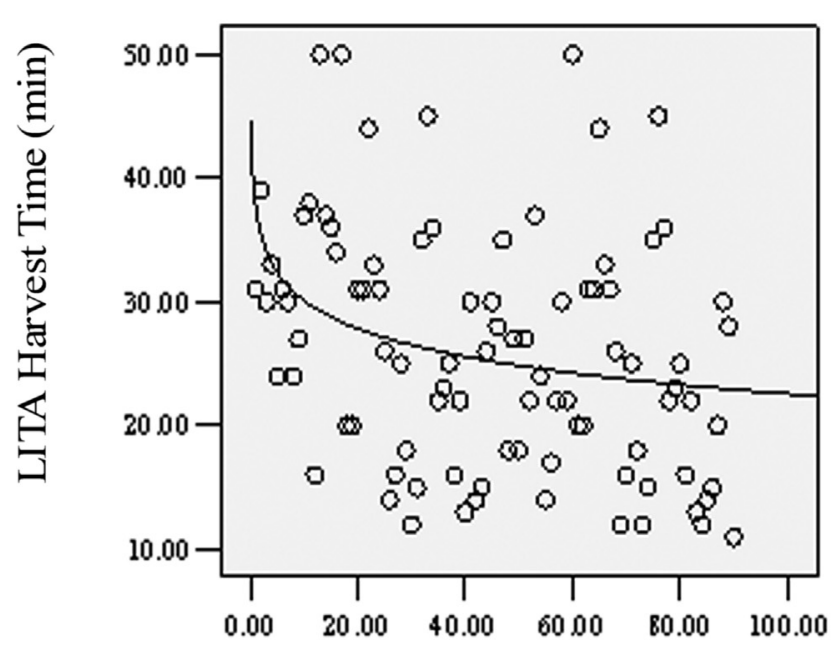

A

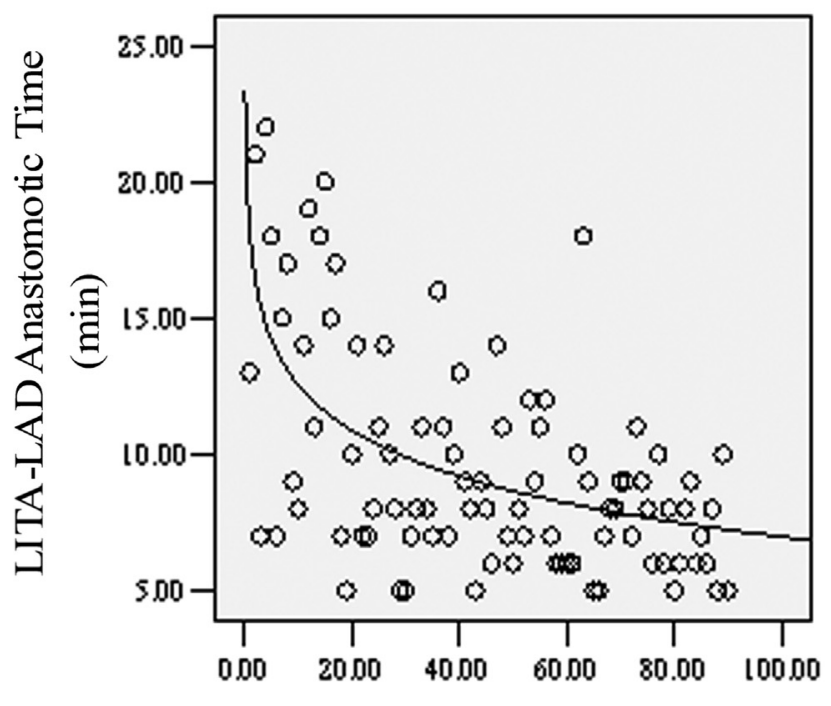

C
Robotic LITA Takedown Number

\section{TECAB Number}
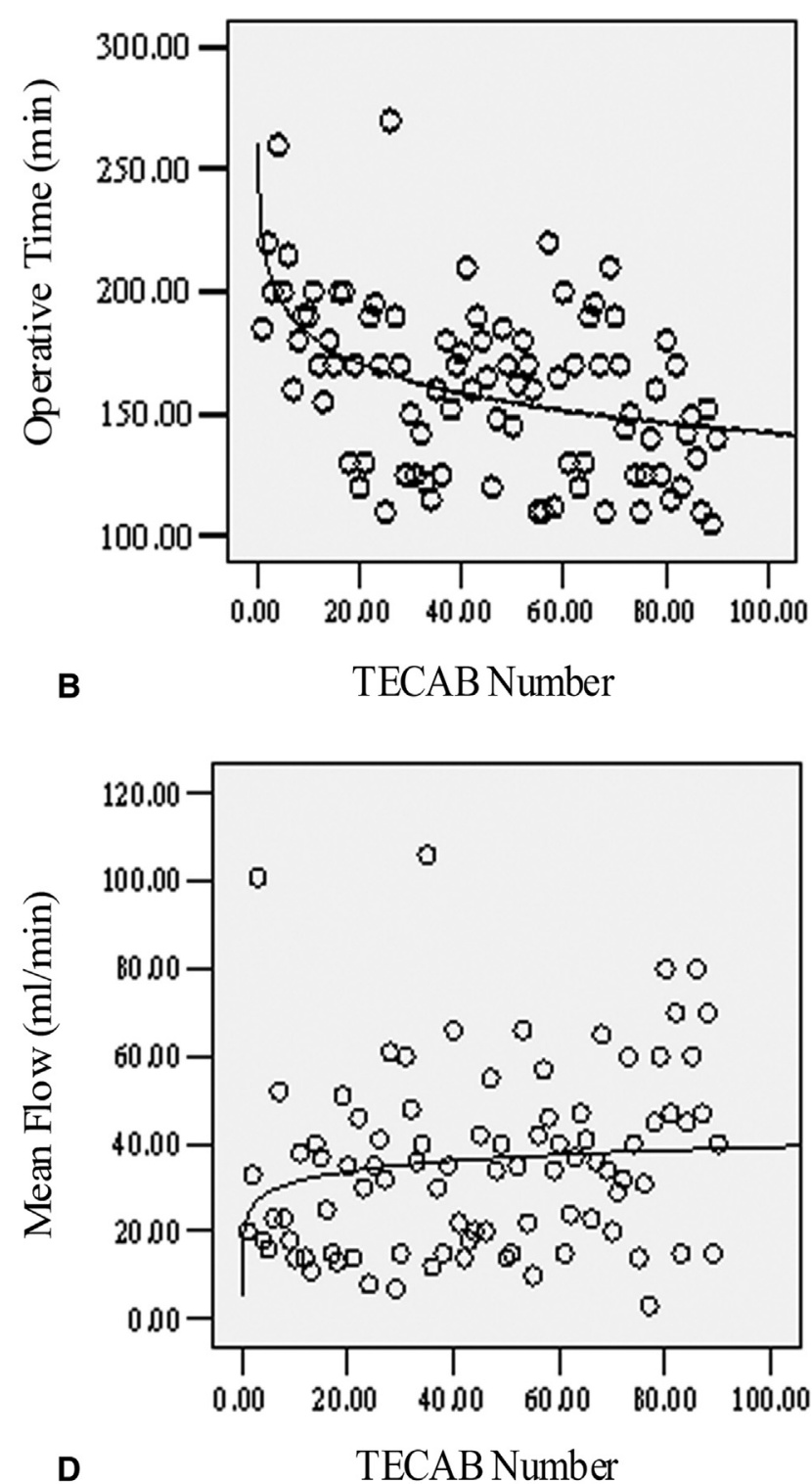

FIGURE 1. A, Learning curve with 230 patients who underwent totally endoscopic coronary artery bypass grafting (TECAB) in terms of the robotic left internal thoracic artery $\left(\right.$ LITA) harvesting time $\left[\mathrm{y}(\mathrm{min})=37-3 \times \ln (\mathrm{x}) ; \mathrm{r}^{2}=0.097, P=.003\right]$, with $\mathrm{y}$ indicating the LITA takedown time and $\mathrm{x}$, the consecutive LITA takedown number. B and C, The learning curves for the 90 patients who underwent TECAB in terms of the operative time $\left[\mathrm{y}(\mathrm{min})=223-17 \times \ln (\mathrm{x}) ; \mathrm{r}^{2}=0.217, P=.000\right]$, and LIMA-LAD anastomotic time $\left[\mathrm{y}(\mathrm{min})=18-2 \times \ln (\mathrm{x}) ; \mathrm{r}^{2}=0.298, P=.000\right]$, with $\mathrm{x}$ indicating the consecutive TECAB number. D, The mean transit blood flow was $35.6 \mathrm{~mL} / \mathrm{min}$, and no significant learning curve was present. $L A D$, Left anterior descending artery.

an infraxiphoid incision because of a diffusely calcified right coronary artery and because the blood flow was not satisfactory after robotic anastomosis of the right internal thoracic artery to the right coronary artery.

A dramatic learning curve was seen for the total operative time of TECAB in our study. Complications such as reoperation for bleeding, stroke, and pneumonia were infrequent in all 3 patient quintiles. Wiedemann and colleagues ${ }^{7}$ analyzed the outcome of 325 patients who had undergone TECAB and found that involvement of the bilateral thoracic arteries and conversion were both risk factors for a prolonged operative time for TECAB. Surgical complications such as anastomotic problems, LITA injuries, and epimyocardial lesions, all of which can increase the conversion rates, can significantly prolong the operative time. Thus, it is very important to make every effort to overcome any surgical problems. The learning curve in our study was truncated because our console surgeon had simultaneously performed $>650$ cases of various types of robotic cardiac surgeries with the same cardiac surgery team. ${ }^{3,4,12}$ Furthermore, the use of port incisions in the left side of the chest without groin incisions and the 
absence of a need for cardiopulmonary bypass greatly optimized the procedure and shortened the learning curve.

Bonaros and colleagues ${ }^{5}$ found that the EuroSCORE was the only independent risk factor for safety in the outcomes of 500 patients who underwent TECAB, underlining the importance of patient selection in the TECAB procedure. High-risk patients cannot tolerate longer operative times and single-lung ventilation, and the consequences in such situations might outweigh the benefits of TECAB. Thus, a careful risk/benefit analysis should be performed during preparation, and patients with low physical reserves might not be suitable for minimally invasive cardiac surgery.

In our series, we found that both LITA preparation without graft injury and completion of the anastomosis on the $\mathrm{BH}$ were the most demanding parts of TECAB. In minimally invasive direct coronary artery bypass grafting with single LITA-to-LAD anastomosis, the graft occlusion rate was reported to be $6 \%$, and anastomotic stenosis of $>50 \%$ was found in $10 \%$ of the patients. ${ }^{13}$ Therefore, meticulous attention to every detail is necessary during the TECAB procedure. Interrupted anastomosis with U-Clips can reduce the possibility of purse-stringing a continuous suture and help to manage the inherent problem of the lack of tactile feedback associated with the da Vinci Surgery System. ${ }^{14}$ For improved hemostasis, we would also suggest large bites with the U-Clips from the outside of the incision of the target vessel. In addition, preconditioning ischemia would help to facilitate a longer anastomotic time with $<80 \%$ to $90 \%$ stenosis in a large proximal LAD. ${ }^{2}$

\section{Study Limitations}

Our study was limited by the relatively small and select group of patients included in the performance of TECAB. In addition, complex multivessel procedures were not contained in the learning curve analysis, because these procedures were not routinely performed.

\section{CONCLUSIONS}

We have concluded that TECAB for single-vessel LITA-to-LAD grafting on the $\mathrm{BH}$ can be performed safely with excellent early and midterm patency rates in selected patients. A stable and well-trained robotic cardiac team and an experienced cardiac surgeon can achieve optimal results after a substantial learning curve.

\section{References}

1. Loulmet D, Carpentier A, D'attellis N, Berrebi A, Cardon C, Ponzio O, et al. Endoscopic coronary artery bypass grafting with the aid of robotic assisted instruments. J Thorac Cardiovasc Surg. 1999;118:4-10.

2. Gao C, Yang M, Wu Y, Wang G, Xiao C, Zhao Y, et al. Early and midterm results of totally endoscopic coronary artery bypass grafting on the beating heart. J Thorac Cardiovasc Surg. 2011;142:843-9.

3. Gao C, Yang M, Wang G, Wang J, Xiao C, Wu Y, et al. Totally endoscopic robotic atrial septal defect repair on the beating heart. Heart Surg Forum. 2010;13: E155-8.

4. Gao C, Yang M, Wang G, Xiao C, Wang J, Zhao Y. Totally endoscopic robotic ventricular septal defect repair in the adult. J Thorac Cardiovasc Surg. 2012; 144:1404-7.

5. Bonaros N, Schachner T, Lehr E, Kofler M, Wiedemann D, Hong P, et al. Five hundred cases of robotic totally endoscopic coronary artery bypass grafting: predictors of success and safety. Ann Thorac Surg. 2013;95:803-12.

6. Holzhey DM, Jacobs S, Walther T, Mochalski M, Mohr FW, Falk V. Cumulative sum failure analysis for eight surgeons performing minimally invasive direct coronary artery bypass. J Thorac Cardiovasc Surg. 2007;134: 663-9.

7. Wiedemann D, Bonaros N, Schachner T, Weidinger F, Lehr EJ, Vesely M, et al. Surgical problems and complex procedures: issues for operative time in robotic totally endoscopic coronary artery bypass grafting. J Thorac Cardiovasc Surg. 2012;143:639-47.e632.

8. Srivastava S, Barrera R, Quismundo S. One hundred sixty-four consecutive beating heart totally endoscopic coronary artery bypass cases without intraoperative conversion. Ann Thorac Surg. 2012;94:1463-8.

9. Schachner T, Bonaros N, Wiedemann D, Lehr EJ, Weidinger F, Feuchtner G, et al. Predictors, causes, and consequences of conversions in robotically enhanced totally endoscopic coronary artery bypass graft surgery. Ann Thorac Surg. 2011;91:647-53

10. Oehlinger A, Bonaros N, Schachner T, Ruetzler E, Friedrich G, Laufer G, et al. Robotic endoscopic left internal mammary artery harvesting: what have we learned after 100 cases. Ann Thorac Surg. 2007;83:1030-4.

11. Bonatti J, Schachner T, Bonaros N, Ohlinger A, Danzmayr M, Jonetzko P, et al. Technical challenges in totally endoscopic robotic coronary artery bypass grafting. J Thorac Cardiovasc Surg. 2006;131:146-53.

12. Gao C, Yang M, Wang G, Wang J, Xiao C, Wu Y, et al. Excision of atrial myxoma using robotic technology. J Thorac Cardiovasc Surg. 2010;139: $1282-5$.

13. Moussa I, Oetgen M, Subramanian V, Kobayashi Y, Patel N, Moses J. Frequency of early occlusion and stenosis in bypass grafts after minimally invasive direct coronary arterial bypass surgery. Am J Cardiol. 2001;88:311-3.

14. Srivastava S, Gadasalli S, Agusala M, Kolluru R, Barrera R, Quismundo S, et al. Beating heart totally endoscopic coronary artery bypass. Ann Thorac Surg. 2010; 89:1873-9; discussion 1879-80. 